\title{
Metodologías de diseño para la innovación y su relación con la competitividad empresarial
}

Luis Aníbal Álvarez Barragán Institución Universitaria Politécnico Gran Colombiano Colombia

María Eugenia Morales Sierra Institución Universitaria Politécnico Gran Colombiano 


\title{
Metodologías de diseño para la innovación y su relación con la competitividad empresarial
}

\author{
Luis Aníbal Álvarez Barragán D , María Eugenia Morales Sierra
}

Institución Universitaria Politécnico Gran Colombiano - Colombia

Para citaciones: Álvarez Barragán, L., \& Morales Sierra, M. (2021). Metodologías de diseño para la innovación y su relación con la competitividad empresarial. Panorama

Económico, 29(1), 73-83.

Recibido: 6 de septiembre de 2020

Aprobado: 30 de noviembre de 2020

Autor de correspondencia:

Luis Aníbal Álvarez Barragán

alvarezla@poligran.edu.co

Editor: Andrés Escobar E. Universidad de Cartagena-Colombia.

Tipología IBN Publindex:

Artículo Resultado de Investigación

Científica y Tecnológica

Copyright: (C) 2021. Álvarez Barragán, L., \& Morales Sierra, M. Este es un artículo de acceso abierto, distribuido bajo los términos de la licencia https://creativecommons.org/licenses/bync-sa/4.0/ la cual permite el uso sin restricciones, distribución y reproducción en cualquier medio, siempre y cuando que el original, el autor y la fuente sean acreditados.

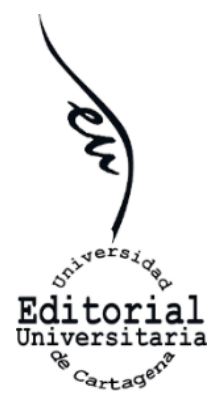

\section{RESUMEN}

La globalización y la libertad de mercados son aspectos relevantes para la toma de decisiones de las naciones y su aparato productivo, donde la innovación es el principal componente para estimular la competitividad y alcanzar los objetivos de desarrollo sostenible. Este artículo analiza las metodologías de diseño para la innovación y su relación con la competitividad de las empresas del sector textil y de las confecciones de Medellín. El objetivo del estudio es describir y explicar la relación que existe entre las metodologías de diseño para la innovación y la competitividad de las pequeñas y medianas empresas del sector textil y de las confecciones de Medellín y su alcance en los resultados económicos. Para efectos metodológicos se usa un modelo de elección discreta y representativa sobre una muestra de pequeñas y medianas empresas del sector textil de Medellín, en las cuales se aplica un instrumento tipo encuesta, diseñado y probado para alcanzar los propósitos de gestión de la información. La investigación tiene un enfoque mixto, alcance descriptivo-explicativo, creando la necesidad de estudiar la aplicación de metodologías de diseño para la generación de innovación empresarial. Los hallazgos del estudio señalan que existe una relación del $64.3 \%$ entre las de metodologías de diseño para la innovación y la competitividad de las empresas que hacen parte del análisis, a su vez, una relación del 62.05\% entre las metodologías de diseño para la innovación y los resultados de la actividad empresarial expresada en términos de ventas, participación de mercado, rentabilidad e imagen corporativa.

Palabras clave: Metodologías de diseño, Pensamiento de diseño, Competitividad, innovación

\section{Design methodologies for innovation and their relationship with business competitiveness}

\begin{abstract}
Globalization and free markets are relevant aspects for the decision-making of nations and their productive apparatus, where innovation is the main component to stimulate competitiveness and achieve sustainable development goals. This article analyses design methodologies for innovation and their relationship with the competitiveness of companies in the textile and clothing sector in Medellín. The objective of the study is to describe and explain the relationship that exists between design methodologies for innovation and the competitiveness of small and medium-sized companies in the textile and clothing sector in Medellín and its scope in economic results. For methodological purposes, a discrete and representative election model is used on a sample of small and medium-sized companies in the textile sector of Medellín, in which a survey-type instrument is applied, designed and tested to achieve the purposes of information management. The research has a mixed approach, descriptive explanatory, creating the need to study the application of design methodologies for the generation of business innovation. The study findings indicate that there is a 64.3 relationship percentage between design methodologies for innovation and the competitiveness of the companies that are part of this analysis, and a 62.05 relationship percentage between design methodologies for innovation and the results of the business activity expressed in terms of sales, market share, profitability and corporate image.
\end{abstract}


Keywords: Design methodologies, Design thinking, Competitiveness, innovation.

\section{INTRODUCCIÓN}

La globalización y el libre intercambio de productos y servicios entre países, representa un gran desafío para las naciones y sus aparatos productivos, por la necesidad de crear andamiajes competitivos, que a través de mejores prácticas, estimulen el desarrollo de productos y servicios que logren satisfacer las demandas y necesidades de los cada día más experimentados y sofisticados usuarios, y asegurar su preferencia y consumo permanente. La competitividad fruto de la innovación, es una de las grandes prioridades de los gobiernos y las empresas para alcanzar nuevos territorios y ampliar los intercambios comerciales, ya que de esta capacidad, depende el desempeño de los productos y su éxito en los escenarios comerciales. La innovación es un componente esencial en este propósito de adquirir mayor capacidad productiva, y de desarrollo de nuevos productos y servicios, y a su vez es una fuente de avance y desarrollo. Las empresas y las naciones que innovan continuamente logran conservar su fortaleza económica (Shepherd, C. Ahmed, P. K., Ramos, L., \& Ramos, C. p, 4 (2019). Innovar significa generar cambios, hacer cosas nuevas, dar nuevas perspectivas, mejorar el desempeño de los útiles organizacionales. Para Drucker (1954), citado por Barbas (2013) y Barbas (2013) la innovación debe ser el resultado de la implementación de metodologías que logren de manera eficiente la transformación de productos para la comercialización e inmersión en un mercado foco, de tal forma que se garantice mayores niveles de competitividad.

Pero esta acción de innovar, no puede ser vista como el fruto de un chispazo creativo, o el seguimiento a tendencias que deben ser incorporadas en los procesos, o tal ves lo que el empresario observó en una feria empresarial y que debe seguir a cabalidad porque así lo demanda la industria. La innovación debe ser un proceso riguroso de diseño; una metodología en donde la creatividad, la experiencia de los diseñadores y el conocimiento del cliente, converjan para el hallazgo de nuevas alternativas y prototipos, que satisfagan los deseos y necesidades de los consumidores. La aplicación de metodologías para el diseño y el uso de servicios profesionales de diseño, garantizan un numero importante de innovaciones que aseguran el crecimiento y la permanencia de las empresas en los territorios donde ejercen funciones y estimulan al desarrollo social y económico de los países, por la supuesta reducción de brechas comerciales, y la diferencia en la balanza comercial, que por muchos años ha dado como ganador al producto foráneo sobre el producto local.

En ese sentido, las metodologías de diseño como el Design Thinking (pensamiento de diseño), se convierten en un aliado importante para las empresas en el propósito de alcanzar ventaja competitiva; al respecto, Porter (1990) advierte, que las ventajas competitivas se logran con innovación en un amplio sentido de la palabra, incluyendo nuevas tecnologías, tendencias y metodologías que como el Design Thinking (pensamiento de diseño), soportan el desarrollo de nuevos productos, servicios y procesos, para lograr una mejora importante en los niveles de productividad, eficiencia y gestión administrativa. En ese mismo orden, Manzano (2008) afirma, que la innovación 
por sí misma no garantiza la competitividad, y es necesario acompañarla de un proceso que asegure un análisis detallado de los factores que intervienen en la práctica de innovar y de las variables que pueden incidir positiva o negativamente en los resultados comerciales y operativos. Es la innovación por diseño entonces, un camino expedito a la solución de problemas, que surgen alrededor de la organización, el cliente y el producto, para alcanzar amplios niveles de competitividad.

El diseño en sí, es uno de los métodos más eficaces, utilizados para lograr desarrollar prototipos de productos, servicios o modelos de negocio, que luego son probados en un mercado meta, no obstante, es muy importante contar con el beneplácito del consumidor; lo cual plantea, el uso de una metodología como el pensamiento de diseño que acerca al cliente y sus necesidades al producto final y no al revés; el producto al cliente. Gaynor (2002).

El diseño en la actualidad da validez a múltiples prácticas profesionales y de gestión del conocimiento, que en su mayoría evitan sesgarse al producto y tomarlo como epicentro del proceso creativo-innovador, y se focalizan en los resultados que el diseño genera en un determinado grupo social, contemplando con visión sistémica todos los componentes de la cadena de valor, Arias et al (2013). En ese mismo orden, Rodríguez \& Rodríguez (2013) proponen, que el Design Thinking es un facilitador de la Innovación. Permite generar innovación y transformación de manera más rápida y más efectiva. Pero además es el proceso metodológico del Design Thinking el que nos permitirá definir en qué debemos innovar; ya que, ayuda a generar ideas de manera rápida y eficiente, sea en el desarrollo de productos, servicios, procesos internos de una empresa u organización, creando formas de comunicación entre el empresario y el cliente o reinventando los modelos de negocio.

Por otra parte, esta técnica de solución de problemas y creación de estrategias se diferencia de otras al vincular el pensamiento creativo (lado derecho del cerebro) con el pensamiento analítico (lado izquierdo del cerebro), sin priorizar un método de pensamiento por sobre el otro, sino que mezcla los aspectos positivos de ambos Rodríguez \& Rodríguez (2013). El pensamiento en diseño lleva a los diseñadores de la divergencia a la convergencia, pasando por una serie de fases que logran empatía absoluta con el cliente para definir como satisfacer sus necesidades y expectativas, definir la problemática principal en diversos contextos, realizar proceso de ideación con base en las oportunidades, prototipar y comercializar las innovaciones. Este proceso posee siete etapas: "definir, investigar, idear, prototipar, seleccionar, implementar y aprender. En el marco de estos siete pasos, se pueden definir problemas, realizar las preguntas más adecuadas, se pueden crear más ideas, y se pueden seleccionar las mejores respuestas". Rodríguez \& Rodríguez (2013).

En Colombia, las empresas han entendido la innovación como parte del proceso que permite avanzar, generar cambios y lograr ventajas competitivas, y el diseño con enfoque cliente centrista, como una metodología que aporta en la construcción y desarrollo de nuevos productos y servicios. No obstante, el informe estructuración del sistema nacional de diseño (2009), reconoce que, si bien es cierto que en Colombia el diseño como soporte a la innovación, disfruta de un alto reconocimiento entre los agentes del gobierno y el aparato productivo; no se han formulado las bases suficientes para su inmersión en las 
empresas, ya que no se ha comprendido a cabalidad su gran contribución al desarrollo empresarial del país. Tan solo en los últimos años según la misma publicación, el diseño se ha dejado de considerar como un gasto innecesario añadido a la producción. Bernal \& Frost (2015) agregan, que el diseño como una estrategia para la competitividad es poco utilizada por las empresas, por ende, la innovación no está soportada por este concepto, y sigue siendo una tarea por cumplir por parte de los empresarios colombianos; siendo esto, uno de los principales factores que amplían las brechas competitivas entre los países que lideran el ranking de la competitividad mundial y los que no, entre los que se encuentran Colombia y sus países vecinos.

Por tal razón, es importante difundir entre los empresarios colombianos, que el diseño y la metodología que lo acompaña; el Design Thinking o pensamiento en diseño, sirven como guía procedimental, en la construcción eficiente de una estrategia de alto rigor intelectual, con alto nivel de creatividad, que de manera holística y sistemática, de forma a ideas que se convierten en propuestas prácticas y atractivas para los usuarios, Arias, Bruera, Cejas, Donato, Mastroscello, Offenhenden \& Sanguinetti (2013), y que ha sido probada con éxito en múltiples organizaciones en países industrialmente desarrollados.

Estudios previos desarrollados en Europa y en especial en la provincia de Cataluña en España por el CDO' (2010), afirman que el diseño como enfoque de la innovación es uno de los elementos predominantes en los planteamientos estratégicos de las empresas y que 9 de cada 10 organizaciones promueven el uso del diseño como una estrategia permanente para lograr amplios niveles de competitividad y eficiencia en la gestión empresarial. Por otro lado, los mismos estudios demuestran que el $80 \%$ de las empresas investigadas han contratado al menos una vez profesionales en diseño y que un 55\% de las mismas, cuenta con un departamento organizado de diseño y diseñadores, que fortalecen los esfuerzos en materia de innovación y desarrollo de nuevos productos.

De la misma forma, un estudio realizado en empresas de Bogotá por Bernal (2016), concluye que, en Colombia y en especial la ciudad de Bogotá, el diseño como una estrategia para la competitividad, es poco utilizada, no obstante, señala que el 83.8\% de los empresarios encuestados contemplarían o verían pertinente el uso permanente del diseño, y que incluso el $49.2 \%$ de las empresas cuentan con un departamento de diseño que constantemente ejecuta labores en beneficio de la artística de los productos y su imagen. Lo cual presume que los empresarios nacionales ven el diseño desde lo puramente estético, y no desde el método que plantea el diseño como una herramienta para el análisis profundo de cada uno de los elementos, que dan origen a la transformación de una idea en un producto terminado, y por consecuencia lograr los niveles adecuados de innovación y competitividad.

En consecuencia, el presente artículo describe y explica la relación que existe entre las metodologías de diseño para la innovación- Design Thinking (pensamiento de diseño) y la competitividad de las empresas Pymes del sector textil y de las confecciones de la ciudad de Medellín, como un acercamiento a la solución de problemas que sufre el empresariado de la región, alrededor de la innovación y la competitividad organizacional, y que ha sido una constante preocupación para el gobierno regional y nacional, en pro

${ }^{1}$ CDO: Barcelona Centre de Diseeny, Centro de diseño de Barcelona 
de mejorar la capacidad competitiva, ya que de ello depende una mejora en los indicadores de productividad agregada y el alcance de los objetivos de desarrollo sostenible propuestos para la nación en la presente década.

\section{METODOLOGIA}

Para la elaboración del trabajo investigativo se tuvo como insumo principal la aplicación de un instrumento tipo encuesta, la cual fue diseñada y probada por Bernal (2016), en estudio similar realizado en la ciudad de Bogotá con empresas de diversas industrias.

Las preguntas propuestas en el instrumento fueron reagrupadas en ocho dimensiones: cultura de innovación, innovación con base en el diseño, competitividad, innovación en productos y procesos, innovación organizacional, innovación de las empresas, innovación con base en el diseño (apropiación), y resultados de la actividad empresarial. Lo anterior, para un mejor análisis de los datos, una vez agregados los diferentes ítems a cada dimensión, y construida la variable que representa la dimensión específica, se procedió a calcular los coeficientes de correlación respectivos entre cada par de dimensiones analizadas a través del programa estadístico SPSS.

Tabla 1. Dimensiones codificadas del instrumento

\begin{tabular}{lll}
\hline Dimensión & Codificación & Items \\
\hline Cultura de Innovación & CEIN & $1-6$ \\
Innovación con Base en el Diseño 1 & ICBENDIS1 & $7.7 \mathrm{~b} .7 \mathrm{c} .7 \mathrm{~d}$ y 8 \\
Competitividad & COMP & $9.9 \mathrm{i}-9 \mathrm{k}$ \\
Innovación en los productos y procesos & IEPYP & $10 .-16$ \\
Innovación Organizacional & INNORGA & $17.17 \mathrm{a}-17 \mathrm{j}$ \\
Innovación de las Empresas & INNDLEMP & 18. 18a.-18j \\
Innovación con Base en el Diseño 2 & ICBENDIS2 & 19. 19a.-19d \\
(Apropiación del diseño) & & 20.20a.-20e. y 21 \\
Resultados de la Actividad Empresarial & RDLAEMP & \\
\hline
\end{tabular}

Fuente: Elaboración propia

Para establecer la relación o la incidencia de variables objeto del estudio, se hizo uso del cálculo de coeficientes de correlación, los cuales permitieron evidenciar la relación que existe entre 2 variables dados sus valores; es decir, el nivel de incidencia que posee una sobre otra (Triola, 2013).

Un estadístico asociado con el coeficiente de correlación es el denominado coeficiente de determinación, el cual se define formalmente como: "...el cuadrado del coeficiente de correlación; su valor, representa la proporción de la variación de la variable dependiente Y que se explica por la variable independiente X" (Bernal, 2016).

Para determinar los niveles de correlación y determinación entre variables objeto de estudio en la presente investigación, y por ser éstas medidas en una escala ordinal, se hizo uso del coeficiente de correlación de rangos de Spearman- $\rho$ (rho de Spearman) y su cuadrado, el coeficiente de determinación, que a su vez mide o explica el grado de correlación y determinación, respectivamente, entre las variables objeto de estudio. 
El coeficiente de correlación de rangos de Spearman es adecuado cuando la escala de medición de las variables está expresada en intervalos, o bien dicha escala puede ser expresada de una forma más adecuada en forma de ordenaciones.

Como su equivalente paramétrico, el coeficiente de correlación producto-momento de Pearson, el coeficiente de correlación de rangos de Spearman también cuantifica el grado de asociación entre dos variables, con la diferencia que permite prescindir de las limitaciones que son impuestas para la aplicación de las pruebas estadísticas paramétricas; por ejemplo, el supuesto de Normalidad (Moore, 2000).

\section{DATOS}

Para determinar la muestra se tomó como marco muestral la base de datos de la Cámara de Comercio de Medellín, donde se identificaron 186 empresas denominadas pequeñas y medianas de diferente naturaleza jurídica dedicadas a la producción textil y las confecciones en general, cuya actividad económica pertenecen a la clase 131. 141. 142. 143.

En concordancia con la clasificación internacional uniforme de todas las actividades económicas ${ }^{2}$ y su año de matrícula y renovación se encuentra entre enero del 2015 y enero del 2019. De allí se tomaron como objeto de estudio 84 empresas y un número idéntico de sus directivos.

\section{Hipótesis}

H 1. Existe relación entre la innovación con base en el diseño y la competitividad de las empresas estudiadas, en aspectos tales como: la productividad, rentabilidad, eficiencia, calidad de los productos, cantidad de nuevos productos, cantidad de productos mejorados, Satisfacción del cliente, mejores prácticas, maquinaria y equipo y tamaño de la empresa.

H 2. Existe relación entre la innovación basada en el diseño y los resultados de la operación tales como las ventas, la participación en el mercado, la rentabilidad y la imagen corporativa.

\section{RESULTADOS}

El gráfico 1 presenta la matriz de correlaciones entre las variables (dimensiones) objeto de estudio, la parte superior de cada una de las casillas representa el coeficiente de correlación entre las variables y la parte inferior de las casillas, representa el nivel de significancia exacto o valor $p$ de la prueba que, según se explicó en el apartado anterior, permitirá concluir si la relación entre dichas variables (dimensiones), resulta ser estadísticamente significativa y por lo tanto extrapolada a la población objeto de estudio.

${ }^{2}$ DANE: Departamento Administrativo Nacional de Estadística. 
Gráfico 1. Coeficientes de correlación de rangos de Spearman (valores P entre paréntesis)

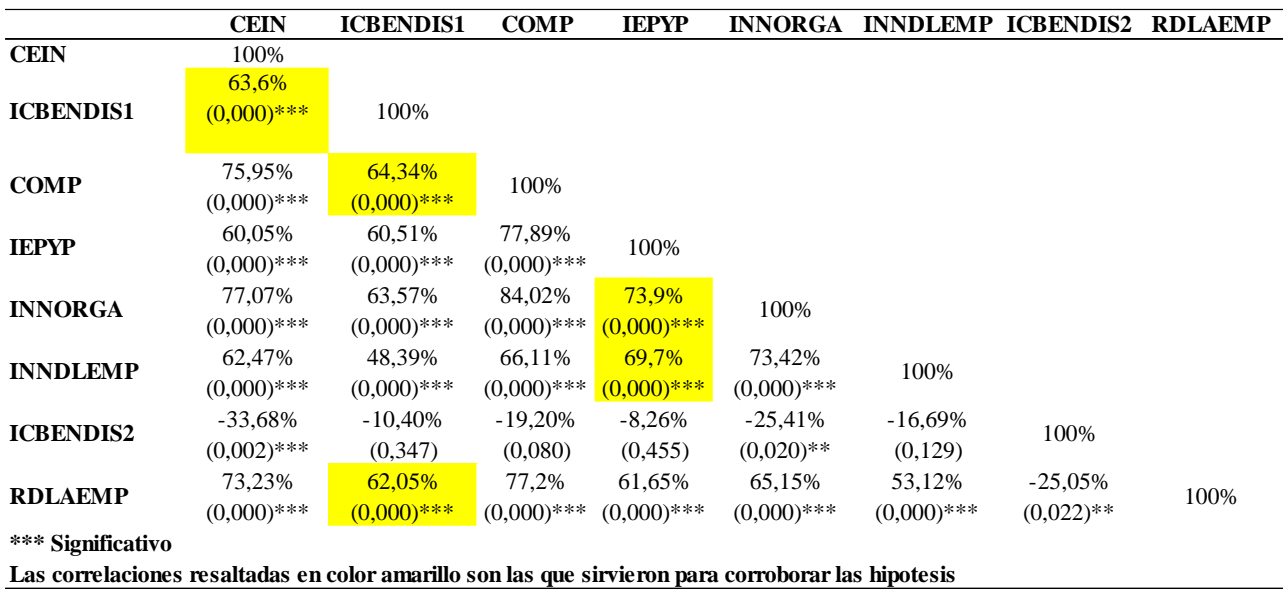

Fuente: Elaboración propia.

Se procederá a calcular los coeficientes de determinación respectivos, para aquellas relaciones que resultan ser estadísticamente significativas (Gráfico 2).

Gráfico 2. Coeficientes de determinación de variables

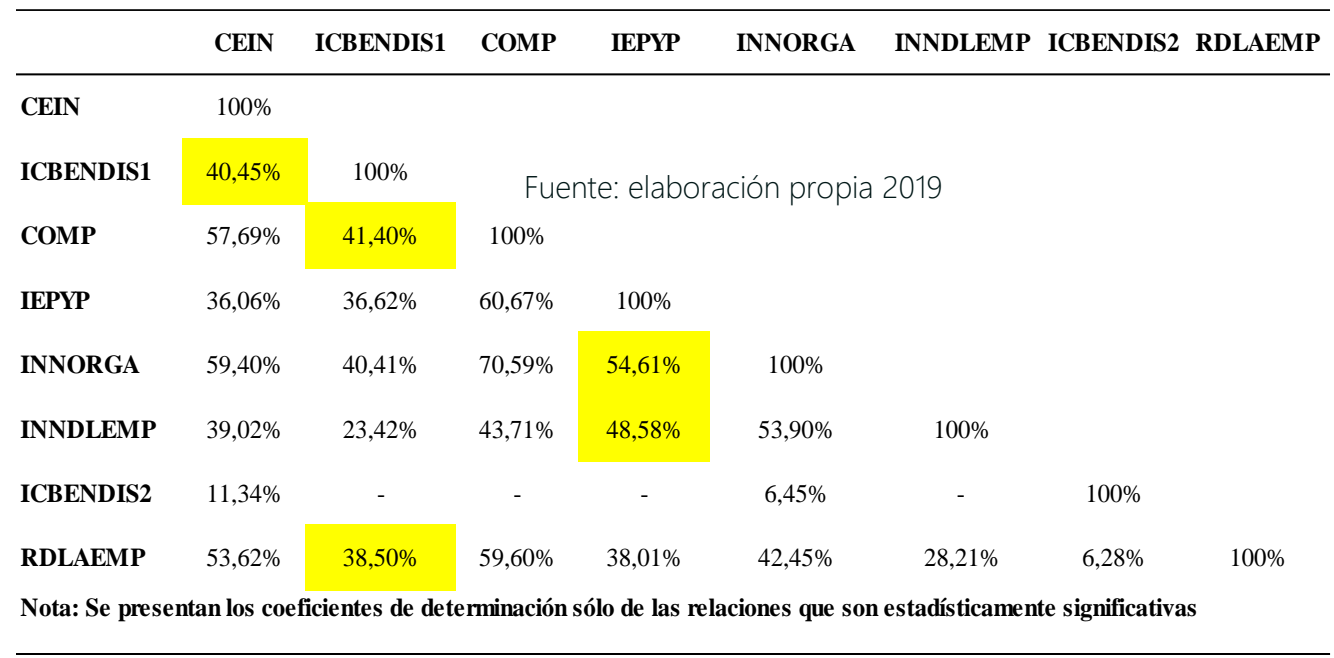

Para el cumplimiento de los objetivos planteados en el estudio, se procede a evaluar los resultados obtenidos entre la relación de las dimensiones ICBENDIS1 (innovación con base en el diseño1- Variable independiente y la COMP (competitividad- variable dependiente); las cuales resultan ser estadísticamente significativas y su grado de determinación. Así mismo se procede a evaluar los resultados obtenidos entre la relación de las dimensiones ICBENDIS1 (innovación con base en el diseño1- Variable independiente y la RDLAEMP (resultados de la actividad empresarial- variable dependiente); las cuales resultan ser estadísticamente significativas y su grado de determinación.

A partir de los resultados presentados en los gráficos 1 y 2 y, se observa una relación positiva fuerte (64.34\%), y estadísticamente significativa entre las dos dimensiones; ICBENDIS1 - innovación con base en el diseño1) y la COMP - competitividad (Valor P: 
0.000). Si se quisiera predecir la competitividad (variable Dependiente o explicada), a partir de los resultados de la innovación con base en el diseño (variable independiente o explicativa), se pudo establecer, que el $41.40 \%$ de la variabilidad en los resultados de la competitividad de las empresas, es explicada por la innovación con base en el diseño. Este resultado es bastante significativo, máxime si se tiene en cuenta que dicha dimensión (competitividad), también debería ser explicada por una mayor cantidad de dimensiones, siendo fundamental el aporte de la innovación con base en el diseño.

De igual forma, a partir de los resultados obtenidos se estableció una relación positiva moderada (62.05\%), y estadísticamente significativa entre las dos dimensiones ICBENDIS1 - innovación con base en el diseño1 y la RDLAEMP - resultados de la actividad empresarial (Valor P: 0.000). Si se quisiera predecir el resultado operacional expresado en términos de ventas, participación de mercado, rentabilidad e imagen corporativa (variable Dependiente o explicada), a partir de la aplicación de la innovación con base en el diseño (variable independiente o explicativa), se podría afirmar que el 38.50\% de la variabilidad en los resultados operacionales de las empresas, es explicada por la innovación con base en el diseño.

Así mismo, ante la pregunta ¿En esta empresa se requiere de forma inmediata los servicios profesionales de diseño?; los resultados obtenidos logran establecer que el $59.5 \%$ de los ejecutivos encuestados se declaran totalmente de acuerdo con el hecho de que requieren de forma inmediata servicios profesionales de diseño para seguir creciendo en su intención de innovar para mejorar su capacidad productiva y competitiva. Lo cual abre una puerta importante para que los profesionales de diseño ofrezcan sus servicios.

Tabla 2. ¿En esta empresa se requiere de forma inmediata los servicios profesionales de diseño?

\begin{tabular}{lrrrr}
\hline & Frecuencia & Porcentaje & $\begin{array}{c}\text { Porcentaje } \\
\text { válido }\end{array}$ & $\begin{array}{c}\text { Porcentaje } \\
\text { acumulado }\end{array}$ \\
\hline Totalmente en desacuerdo & 12 & 14.3 & 14.3 & 14.3 \\
\hline En desacuerdo & 11 & 13.1 & 13.1 & 27.4 \\
\hline Indiferente & 6 & 7.1 & 7.1 & 34.5 \\
\hline De acuerdo & 5 & 6 & 6 & 40.5 \\
\hline Totalmente de acuerdo & 50 & 59.5 & 59.5 & 100 \\
\hline Total & 84 & 100 & 100 & \\
\hline
\end{tabular}

Fuente: Elaboración propia

\section{DISCUSIÓN DE RESULTADOS}

Desde una postura crítica y reflexiva se asume que las metodologías de diseño en concordancia con lo expuesto por Porter (1990) y los hallazgos del presente estudio, son gestoras de competitividad en un amplio sentido de la palabra, y aportan a la innovación y la transformación eficiente de los productos de cara a la comercialización e inmersión en un mercado foco. Así mismo, los resultados del estudio permiten comprobar la hipótesis de que existe una estrecha correlación entre la innovación con base en el diseño y la competitividad de las empresas evaluadas en un $64.34 \%$, y que solo la variable innovación con base en el diseño, tiene una incidencia directa o determinante del 41.40\% en la competitividad y los resultados en general. Vale la pena resaltar que la 
competitividad empresarial debe ser explicada por una suma de variables y sorprende que para la industria textil y de las confecciones de Medellín una sola variable (la innovación con base en el diseño), justifica los resultados de competitividad organizacional. Esto, debe generar consciencia en los empresarios de la región sobre la importancia de innovar fundamentados en metodologías de alto rigor intelectual que estimulan con enfoque cliente centrista el desarrollo de productos y servicios que garantizan la permanencia de las empresas en sus mercados con altos niveles de excelencia organizacional.

De la misma forma a partir de los resultados del estudio, se logró comprobar la hipótesis de que existe una relación entre la innovación basada en el diseño y la variabilidad de los resultados de la operación tales como las ventas, la participación en el mercado, la rentabilidad y la imagen corporativa en un 38.5\%, considerando que existen muchas variables que podrían afectar directamente dicha variabilidad. Sin embargo, estos resultados son muy subjetivos y dependieron de la percepción de cada uno de los entrevistados. No obstante, la información obtenida no contrasta con lo expuesto por Stamm (2004) quien señala que en estados unidos el 80\% de las empresas más importantes listadas en la revista Fortune, se han apropiado de la innovación por diseño como una estrategia para el desarrollo de nuevos productos y mejores prácticas empresariales, y mas que subjetivo es un resultado tangible en la carrera de las compañías por ganar las batallas de la ventaja competitiva.

Finalmente, a través de los hallazgos obtenidos se logra establecer que el 59.5\% de los ejecutivos encuestados están de acuerdo en torno a la necesidad de contratar servicios profesionales de diseño para mejorar su capacidad productiva y competitiva. Este resultado, se asocia a la investigación realizada por el Banco de Desarrollo de América Latina (2013) en asocio con la Fundación Observatorio Pyme de Buenos Aires Argentina, la cual propone que en América Latina, tan solo el 22\% de las empresas utilizan la innovación con base en el diseño profesional, tanto en la modalidad make como la modalidad (buy-pagada). Que otro 48\%, de las empresas llevan a cabo actividades de diseño al interior (make-hecha al interior) de forma no profesional, en general delegando estas funciones en gerentes o directivos de la compañía. El restante $30 \%$ de las empresas no utiliza ningún servicio de diseño por no considerarlo necesario, demostrando este último resultado que aún queda mucho por trabajar en términos de promoción e inserción del diseño en el proceso productivo. En total, los directivos organizacionales tienen la idea de que la contratación de profesionales en diseño no incide de manera significativa en la rentabilidad de las empresas, aunque sí en la satisfacción del usuario, la imagen corporativa y la de la marca en el mercado. A pesar de ello, la evidencia estadística muestra que las empresas que utilizan servicios profesionales del diseño (22\% del total), tanto bajo la modalidad make (hacer )cómo buy(comprar), muestran un mejor desempeño que el resto de las empresas encuestadas.

\section{CONCLUSIONES}

La innovación puede no estar soportada por metodologías para el diseño en gran parte de las industrias de Bogotá y tal vez del país según lo señala Bernal (2016), pero para la industria textil y de las confecciones de Medellín el panorama es distinto, ya que el 64.34\% de los empresarios evaluados, señalan un uso permanente de las metodologías de diseño 
para generar competitividad organizacional y solo la variable innovación determina un 41.4\% la competitividad y lo resultados generales de la organización.

Así mismo, la innovación con base en el diseño, es responsable en dichas organizaciones del $38.5 \%$ de la variabilidad de los resultados operativos tales como las ventas, la participación de mercado, la rentabilidad y la imagen corporativa. Teniendo en consideración que existen muchas variables en juego que pueden explicar el 100\% de los resultados, solo la variable innovación por diseño logra un papel preponderante en dichas variaciones.

En ese sentido, la innovación con base en metodologías de diseño para alcanzar una mejor posición competitiva de las empresas y mejorar los resultados corporativos es imprescindible, y el llamado a los empresarios del país y al aparato productivo en general, es apropiarse de estas metodologías de diseño para satisfacer sus necesidades de nuevos productos, servicios y procesos, que garanticen un aprovechamiento de los recursos corporativos y los mercados donde realizan operaciones, para aumentar los intercambios y generar mayores ingresos que aseguren la permanencia de las organizaciones en sus territorios, en las condiciones que ofrecen la globalización y el libre mercado.

No basta, que el 59.5\% de los ejecutivos expresen la prioridad en la contratación de servicios profesionales de diseño para mejorar la innovación y alcanzar ventajas competitivas, es necesario que haya confianza en el modelo y esfuerzos desde el punto de vista monetario y entender que estos recursos no pueden ser tipificados como gastos sino como inversión para el progreso empresarial.

Es necesario considerar que el presente estudio tuvo limitaciones en cuanto a su profundidad para llegar a datos más contundentes; ya que, son pocos los estudios y casos al respecto en Colombia y en especial en el sector textil y de las confecciones. De tal forma que se invita a los académicos y/o empresas del sector al desarrollo de nuevos estudios de carácter empírico, que permitan la construcción de casos, donde se involucren empresas de otras ciudades del mismo gremio para contrastar resultados, que conlleven a un conocimiento más detallado de los escenarios reales de la industria y sus prácticas cotidianas de innovación.

Finalmente, se sugiere la elaboración de un análisis profundo del impacto de innovar con base en el diseño en los resultados financieros de las organizaciones, de tal forma que los resultados del estudio, logren concientizar a los empresarios de su apropiación como una estrategia definitiva para lograr la competitividad de cara a superar con mayor eficiencia los retos que demanda la globalización. Es además importante el desarrollo de un software que permita sistematizar a través de matrices, cada uno de los factores y variables de la innovación y la competitividad, de cara a prospectar resultados y minimizar los riesgos y la incertidumbre en la toma de decisiones gerenciales.

\section{REFERENCIAS}

Álvarez, L. Herrera, Y, Fuertes, M. (2017), La innovación con base en el diseño y su relación con la competitividad de las Pymes del sector textil de Medellín, 2016 
Arias, F., Bruera, I., Cejas, C., Donato, V., Mastroscello, L., Offenhenden, C., \& Sanguinetti, M. (2013). Innovación y diseño entre las PyME industriales, Documento No. 9.

Ariza, R., \& Ramírez, R. (2007). Herramientas para mejorar la gestión del diseño en Pymes. 6a Jornadas de Innovación y Desarrollo.

Barbas, E. (2013). Gestionando la innovación. Recuperado de [http://www.enricbarba.com/2011/07/Peter-Drucker-y-la-innovación].

Centro de diseño de Barcelona (2010). El impacto económico de las empresas de Cataluña. Extractado de: http://www.bcd.es/site/unitFiles/2521/Impacte\%20economic CAST.pdf

Bernal, C. Frost, S (2015). Innovación soportada en el diseño en las empresas en la ciudad de Bogotá. Bogotá: Universidad de La Sabana

Bernal, C. (2016). Metodología de la Investigación. 4ta edición. Bogotá D. C.: Pearson.

Brown, T. (2008). Definitions of design Thinking. Design Thinking: Thoughts by Tim Brown, Harvard Business Review, 7, 84-97.

Comisión Nacional del Diseño (2009). Estructuración del sistema nacional del diseño. Ministerio del comercio, Industria y turismo. Bogotá. Extractado de: http://www. mincit.gov.co/publicaciones. php?id=17751

BID (2013). Innovación y diseño entre las Pymes industriales. Serie políticas públicas y trasformación $\begin{array}{llll}\text { productiva } & \text { No. } & 9 . & \text { Recuperado de }\end{array}$ http://publicaciones.caf.com/media/29151/libro pymes bsas web.pdf

Gaynor, G. (2002). Innovation by design: what it takes to keep your company on the cutting edge.

Gutiérrez, J. (2015). ¿Cómo innovar de la mano de Ruta N? Cátedras de innovación empresarial. Medellín: Universidad EAFIT

UNAL (2009). Estudio estratégico y de caracterización del diseño en las MiPymes colombianas. Informe de la Universidad Nacional de Colombia para el Ministerio de Industria y Comercio. Bogotá: UNAL

Martínez, E. (2005). Importancia del diseño industrial en la gestión estratégica de la empresa. Universia Business Review, 8, 52-67.

Manzano, W. (2008). La gestión de la innovación como herramienta para la competitividad.

Morales, L. (2008). Nuevas tendencias en innovación: Design Thinking.

Porter, M. E. (1990). The competitive advantage of nations.

Rodríguez, B. D, Rodríguez, M.A. (2013). Innovación por Desing Thinking. Creatividad para los negocios

Schwab, K., Sala-i-Martin, X. (2016). The Global Competitiveness Índex. Recuperado de https://www. weforum.org/reports/the-global-competitiveness-report-2016-2017-1

Sistema Nacional de Diseño (2009). Estructuración del Sistema Nacional de Diseño. Bogotá D. C.: Universidad Nacional de Colombia.

Shepherd, C. D., Ahmed, P. K., Ramos, L., Ramos, C. (2019). Administración de la innovación.

Stamm, B. (2004). Innovation—What's Design Got to Do with It?. Design Management Review, 15(1), 10-19.

Triola, M. (2013). Estadística, 11a ed. México D. F.: Pearson. 